\title{
Papers
}

\section{Relation between burden of disease and randomised evidence in sub-Saharan Africa: survey of research}

\author{
Petros Isaakidis, George H Swingler, Elizabeth Pienaar, Jimmy Volmink, John P A Ioannidis
}

\begin{abstract}
Objective To evaluate whether the amount of randomised clinical research on various medical conditions is related to the burden of disease and health needs of the local populations in sub-Saharan Africa.

Design Construction and analysis of comprehensive database of randomised controlled trials in sub-Saharan Africa based on Medline, the Cochrane Controlled Trials Register, and several African databases.

Setting Sub-Saharan Africa.

Main outcome measures Number of trials and randomised subjects for each category of disease in the global burden of disease taxonomy; ratios of disability adjusted life years (DALYs) per amount of randomised evidence.

Results 1179 eligible randomised controlled trials were identified. The number of trials published each year increased over time. Almost half of the trials $(\mathrm{n}=565)$ had been done in South Africa. There was relatively good correlation between the estimated burden of disease at year 2000 and the number of trials performed $(r=0.53, \mathrm{P}=0.024)$ and the number of participants randomised $(r=0.68, \mathrm{P}=0.002)$.

However,some conditions-for example, injuries (over 20000 DALYs per patient ever randomised)-were more neglected than others.

Conclusion Despite recent improvements, few clinical trials are done in sub-Saharan Africa. Clinical research in this part of the world should focus more evenly on the major contributors to burden of disease.
\end{abstract}

\section{Introduction}

Demand is increasing for research to be prioritised according to the importance of health issues, ${ }^{1}$ and burden of disease measures have been proposed to aid this process. ${ }^{2}$ There is concern that little medical research is done on diseases affecting people in developing countries. ${ }^{3}$ Sub-Saharan Africa is a developing area facing severe, pressing, and often unique health challenges. ${ }^{4}$ Its burden of disease per million people is estimated to be five times higher than that of established market economies. $^{2}$

Effective interventions are needed to improve health, and efficacy is best assessed by randomised controlled trials. An evaluation of randomised control- led trials could therefore be used to determine the amount of clinical research on different health conditions. Randomised controlled trials conducted in Africa have not been comprehensively assessed, and it is not known whether the trials cover the local health needs. We therefore constructed a comprehensive database of randomised controlled trials in subSaharan Africa over the past 50 years. We then evaluated whether the amount of randomised evidence relates to the burden of different health problems and whether specific conditions are neglected.

\section{Methods}

\section{Eligibility criteria}

We considered all randomised controlled trials conducted in sub-Saharan Africa that investigated one or more health problems. ${ }^{2}$ We excluded nonrandomised and pseudorandomised controlled trials; trials enrolling non-local populations (such as tourists); trials not in humans; and trials studying issues that could not be related to a disease problem or group of problems-for example, measuring physiological variables. Multinational trials qualified if some patients had been recruited in sub-Saharan Africa. Studies in northern Africa were excluded (this region is included in the Middle Eastern crescent in the global burden of disease estimates). ${ }^{2}$ Meeting abstracts, books, and other reports were excluded.

\section{Identification of trials}

We searched sequentially Medline (to February 2000), the Cochrane Controlled Trials Register (issue 2, 2000), and the African Published Trials Register of the South African Cochrane Center. The African register has been developed and continuously updated over the past three years from seven diverse international and African databases plus hand searching of back years from 12 major African journals. Terms reflecting randomised controlled trials were conjugated with "Africa," "sub-Saharan Africa," and specific geographical names. We initially evaluated the abstracts of trials. If the abstract was unavailable, left uncertainty on the study design, or lacked required data, we retrieved the full articles.

\section{Database}

From each article, we extracted the following information: author, journal, year of publication, unit

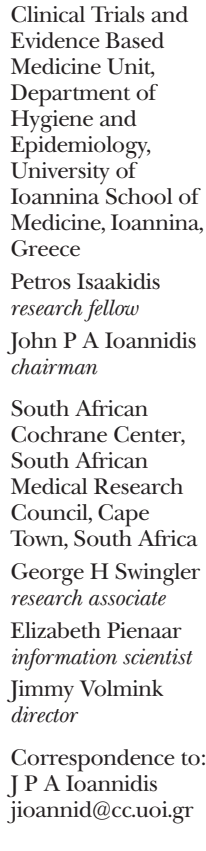

BMJ 2002;324:1-5 
of randomisation (individual or cluster), sample size, disease(s) targeted (and taxonomy in the Global Burden of Disease ${ }^{2}$ ), type of intervention (therapeutic or preventive), and country or countries of recruitment. For studies that also recruited in non-eligible countries, we focused on the number of participants enrolled in subSaharan Africa.

We excluded articles that obviously reflected secondary publications of a main report. Because some secondary publications could be missed, we cross checked every identified article in the database using authors, type of disease(s), and country. Any uncertainty about the primary publication was resolved by discussion among the team. In general, the study with the largest sample size and most clinically important outcomes qualified for primary publication.

\section{Analyses}

Descriptive analyses include the geographical distribution of trials, number of new trials published, and estimates of the number of trials performed per million people in various countries (1990 population estimates). ${ }^{5}$ The main analysis evaluated the number of trials and the number of randomised participants for each category of health problem in the global burden of disease taxonomy. ${ }^{2}$ This taxonomy was developed at the Harvard School of Public Health and the World Health Organization and is recognised worldwide. ${ }^{1267}$ Burden of disease estimates add the number of lost years due to early death plus equivalents of "lost" years due to living with disability from various diseases. The sum is measured in disability adjusted life years (DALYs). For each major disease category we estimated the ratio of burden of disease in each trial and the ratio of burden of disease per participant.

We did separate analyses using either all eligible trials and the estimated burden of disease at 2000 or only trials published before 1990 and the burden of disease at 1990. In sensitivity analyses, we considered only trials with at least 100 randomised subjects or only trials with at least 500 randomised participants, to exclude small trials with limited evidence. For cluster trials, we used the number of participants. Exclusion of cluster trials did not affect the results (data not shown).

We used rank correlation analyses to evaluate whether the number of trials or participants correlated with the DALYs attributed to each health problem. We

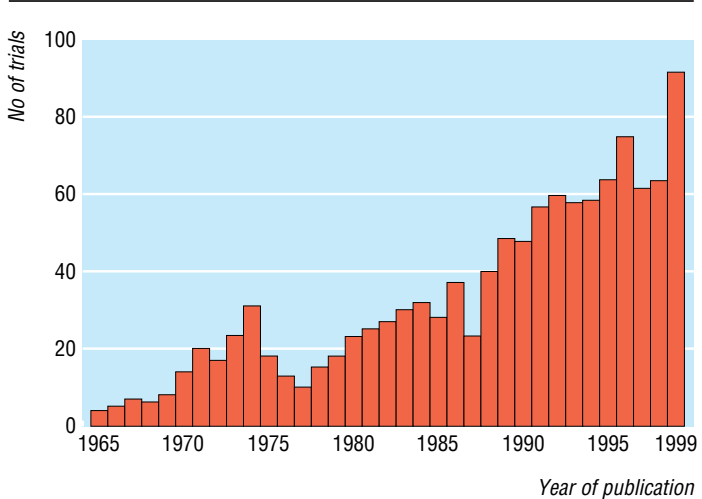

Fig 1 Number of randomised controlled trials in sub-Saharan Africa published during 1965-99. Twelve trials were published during 1949-64 and eight in early 2000 did such analyses for all health problem categories using the key subcategories of the global burden of disease taxonomy for communicable, maternal, perinatal, and nutritional conditions (category I, including subcategories A to E); non-communicable diseases (category II, including subcategories A to N); and injuries (category III). ${ }^{2}$ We also examined separately the various diseases within category IA (infectious and parasitic diseases) as these diseases alone account for half the local burden of disease.

We used SPSS 10.0 for all analyses. P values are two tailed.

\section{Results}

We screened 2803 references (Medline 875, Cochrane Clinical Trials Registry 940, South African Cochrane Center Registry 988) and fully scrutinised 1334 articles; 123 were ineligible (93 publications pertained to other randomised controlled trials, 15 were non-randomised studies, 13 were non-African studies, and two were physiology studies), seven trials gave no sample size, and full articles could not be retrieved for 25 studies of uncertain design. Of the 1179 eligible randomised controlled trials, 93 studied two diseases (and were thus counted for both). A total of 795 trials $(67.4 \%)$ focused on treatment rather than prevention. The median sample size was 90 (interquartile range 42-238). Only 535 trials had over 100 participants; 154 trials had over 500 participants; and 79 trials had over 1000. Cluster trials $(n=60)$ enrolled more participants than trials that randomised individuals (median $742 v$ $82, \mathrm{P}<0.001$ ). The average sample size was bigger in recent trials than older trials (median 63 for trials up to $1990 v 120$ for trials published after 1990, $\mathrm{P}<0.001$ ).

Publication of randomised controlled trials increased over time (fig 1). Ninety two were published in 1999, more than in any previous year. Almost half of the trials had been done in South Africa $(n=565)$. Four other countries accounted for another quarter $($ Nigeria $=98$, Kenya $=89$, Gambia $=56$, Tanzania $=$ 50). Only 19 countries had more than one trial per million population. South Africa accounted for over $90 \%$ of all trials on malignant, respiratory (31.33), digestive $(69 / 76)$, musculoskeletal $(27 / 27)$, and congenital (2/2) diseases and 75-90\% of trials on diabetes (10/13), endocrine (9/10), cardiovascular (75/99), and genitourinary (29/34) diseases and injuries (26/31), but for only $14 \%$ of trials on infectious and parasitic diseases $(74 / 150)$.

Overall, there was good correlation between burden of disease and randomised evidence (table 1) with modest differences depending on how randomised evidence was measured (table 2). However, good correlation does not ensure a proportional relation (fig 2). For the burden of disease per trial performed, the worst ratios were for congenital anomalies, injuries, respiratory infections, and conditions arising during the perinatal period. The best ratios were for oral conditions, diabetes mellitus, musculoskeletal diseases, maternal conditions, and digestive diseases (table 1).

Inequalities were more evident when we considered the burden of disease per participant. For injuries, which are the second largest contributor of DALYs in the area after infectious and parasitic diseases, there were 20000 
Table 1 Burden of disease and evidence from randomised controlled trials for main categories of human disease in sub-Saharan Africa

\begin{tabular}{|c|c|c|c|c|c|}
\hline \multirow[b]{2}{*}{ Disease category } & \multirow{2}{*}{$\begin{array}{l}\text { Burden of disease in } 2000 \\
\text { (1000 DALYs) }\end{array}$} & \multirow{2}{*}{$\begin{array}{c}\text { No of trials (No } \\
\text { before 1990) }\end{array}$} & \multirow{2}{*}{$\begin{array}{c}\text { No of } \\
\text { participants }\end{array}$} & \multicolumn{2}{|c|}{$\begin{array}{c}\text { Ratio of burden of disease (1000 } \\
\text { DALYs) }\end{array}$} \\
\hline & & & & Per trial & Per participant \\
\hline Infectious and parasitic diseases & 131327 & $540(183)$ & 813305 & 243 & 0.16 \\
\hline Respiratory infections & 29077 & $18(7)$ & 52811 & 1615 & 0.55 \\
\hline Maternal conditions & 5691 & $85(30)$ & 25148 & 67 & 0.23 \\
\hline Conditions arising during perinatal period & 18700 & $30(8)$ & 28381 & 623 & 0.66 \\
\hline Nutritional deficiencies & 8389 & $105(37)$ & 111922 & 80 & 0.07 \\
\hline Malignant neoplasms & 8114 & $46(30)$ & 128786 & 176 & 0.06 \\
\hline Diabetes mellitus & 522 & $13(9)$ & 1276 & 40 & 0.41 \\
\hline Endocrine disorders & 1168 & $10(8)$ & 287 & 117 & 4.07 \\
\hline Neuropsychiatric conditions & 15788 & $41(21)$ & 3580 & 385 & 4.41 \\
\hline Sense organ diseases & 2460 & $10(2)$ & 607 & 246 & 4.05 \\
\hline Cardiovascular diseases & 13390 & $99(59)$ & 5648 & 135 & 2.37 \\
\hline Respiratory diseases & 9037 & $33(13)$ & 3320 & 274 & 2.72 \\
\hline Digestive diseases & 5418 & $76(48)$ & 5545 & 71 & 0.98 \\
\hline Genitourinary diseases & 2651 & $34(15)$ & 1903 & 78 & 1.39 \\
\hline Skin diseases & $-^{\star}$ & $6(3)$ & 407 & - & - \\
\hline Musculoskeletal diseases & 1404 & $27(22)$ & 1536 & 52 & 0.91 \\
\hline Congenital anomalies & 5224 & $2(0)$ & 1321 & 2612 & 3.95 \\
\hline Oral conditions & 534 & $18(8)$ & 3567 & 30 & 0.15 \\
\hline Injuries & 58352 & $31(19)$ & 2887 & 1882 & 20.21 \\
\hline
\end{tabular}

*Burden of disease considered small, no exact data given.

Data are not shown for 48 trials (32 published before 1990) that examined topics such as analgesia and anaesthesia that cannot be categorised under the global burden of disease taxonomy.

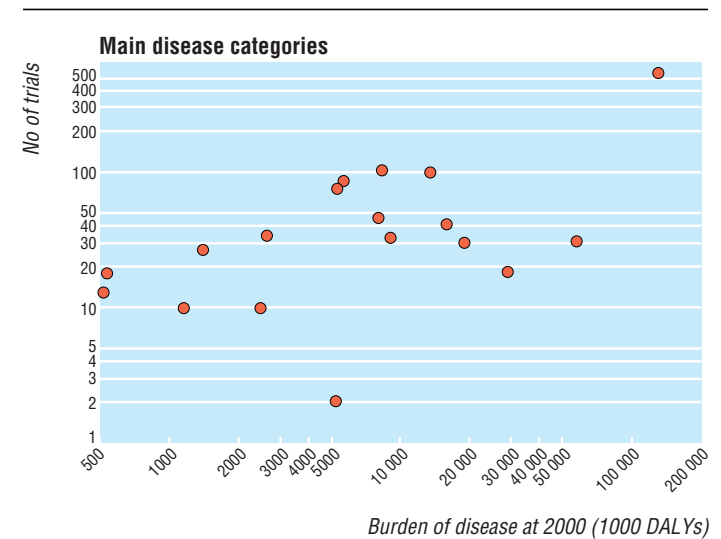

Fig 2 Correlation between burden of disease and number of randomised trials in sub-Saharan Africa for major disease categories defined in global burden of disease taxonomy. Data on skin diseases are not shown as the burden of disease was small and no exact data were given

DALYs per participant. Neuropsychiatric conditions, the fifth cause of DALYs, were also comparatively neglected (4410 DALYs per participant). The ratios were more favourable for the other three major contributors of DALYs (161 DALYs/participant for infectious and parasitic diseases, 551 for respiratory infections, and 659 for conditions arising during the perinatal period). However, the ratio exceeded 150 DALYs per participant for all conditions except nutritional deficiencies and malignant neoplasms.

\section{Infectious and parasitic diseases}

An analysis restricted to infectious and parasitic diseases showed good correlation between the estimated burden of disease and the amount of randomised evidence, with modest differences depending on how randomised evidence was measured (table 2). Despite the good correlation, differences in emphasis were discernible (fig 3). Least attention had been given to diarrhoeal diseases (834000 DALYs per trial), childhood cluster diseases (628 000/ trial), and HIV (571 000/trial). Results were similar when we considered DALYs per participant (data not shown).

\section{Discussion}

We identified just over 1000 randomised controlled trials conducted in sub-Saharan Africa in the past 50 years. This is a small amount of clinical research activity considering the large local burden of disease. Despite an overall good correlation between the amount of randomised research and burden of disease, some conditions have been neglected.

\section{Strengths and weaknesses}

Although the number of trials or enrolled participants is a measure of research activity, it may not reflect the public health emphasis given to a disease. Firstly, trials can vary substantially in size and importance. Nevertheless, analyses limited to studies with over 100 or over 500 participants gave similar results. Secondly, other types of clinical research are also important. However, randomised controlled trials are the stand-

Table 2 Correlation between estimated burden of disease and number of randomised controlled trials and enrolled subjects

\begin{tabular}{|c|c|c|c|c|}
\hline & \multicolumn{2}{|c|}{$\begin{array}{c}\text { All major categories of } \\
\text { disease }\end{array}$} & \multicolumn{2}{|c|}{$\begin{array}{l}\text { Infectious disease } \\
\text { category }\end{array}$} \\
\hline & $r^{*}$ & $P$ value & $r^{*}$ & $P$ value \\
\hline \multicolumn{5}{|l|}{ Burden of disease in 2000 and: } \\
\hline Total No of trials & 0.53 & 0.024 & 0.85 & $<0.001$ \\
\hline No of trials with $n>100$ & 0.64 & 0.004 & 0.79 & 0.001 \\
\hline No of trials with $n>500$ & 0.61 & 0.007 & 0.78 & 0.001 \\
\hline Total No of randomised subjects & 0.68 & 0.002 & 0.25 & 0.39 \\
\hline \multicolumn{5}{|l|}{ Burden of disease in 1990 and: } \\
\hline No of trials before 1990 & 0.35 & 0.15 & 0.67 & 0.009 \\
\hline No of trials before 1990 with $n>100$ & 0.61 & 0.009 & 0.62 & 0.018 \\
\hline
\end{tabular}


ard for therapeutic and preventive interventions, are easy to identify, and provide a measurable surrogate of the intensity of topical clinical research. Thirdly, for some conditions it is more difficult to run trials. For example, war is a major contributor of DALYs in this area. Randomised trials may be almost impossible to implement in war stricken areas, although it is questionable whether medical research is helpless against war and its consequences. ${ }^{8}$ Despite this difficulty, other unintentional injuries account for more DALYs than war and are amenable to preventive interventions. ${ }^{9}$ Finally, publication bias may influence if and where African trials are published. ${ }^{10} 11$ Nevertheless, our search included local journals and it is therefore unlikely that we missed many published trials.

\section{Randomised evidence in other continents}

For many diseases relevant randomised controlled trials may have been done in other continents. Systematic screening of all trials worldwide would have been impracticable. In addition, extrapolation of results from other continents to Africa causes problems because interpretation of generalisability would be subjective and the trials would not take into account the important local challenges that often occur when implementing medical interventions in Africa. ${ }^{12}$ For the three under-researched sections in the infectious diseases category (diarrhoea, childhood diseases, and $\mathrm{HIV}$ ), the issues in Africa are very different from those in the Western world-for example, the interaction of nutrition and diarrhoeal diseases, the logistics of immunisation against childhood diseases, and the infrastructure needed for monitoring highly active antiretroviral therapy (even if these drugs become affordable in Africa).

Almost a million randomised trials have been done worldwide, ${ }^{13}$ and the Cochrane registry already includes over 300000 trials. Most trials have been done in countries with an established market economy. Even if we assume that 150000 trials have been done in such countries (a low estimate), the overall ratio of burden of disease per trial is about 400 times higher in Africa than in established market economies. Estimates are worse if we exclude South Africa, a country with a mixed economic profile.

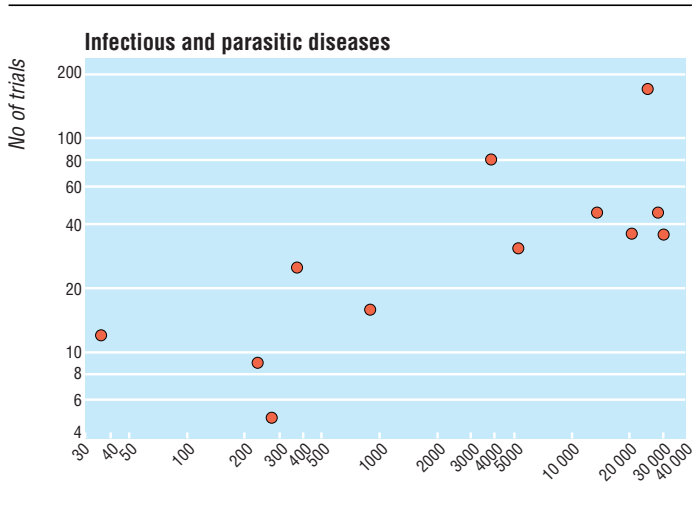

Fig 3 Correlation between burden of disease and number of randomised trials in sub-Saharan Africa for subcategory of infectious and parasitic diseases. Data are not shown for dengue fever (burden of disease 16000 DALYs, no trials) and Japanese encephalitis (no burden of disease or trials)

\section{What is already known on this topic}

Sub-Saharan Africa has a large burden of disease

Little clinical research is conducted for problems affecting sub-Saharan Africa

\section{What this study adds}

Only 1179 randomised controlled trials conducted in sub-Saharan Africa in the past 50 years were identified

Correlation between the amount of randomised evidence and the estimated burden of disease was fairly good

However, some disease categories were more neglected than others, with the worst being injuries

\section{Implications for policymakers and the future}

Most African countries cannot afford to support medical research. ${ }^{14}$ In addition, the pharmaceutical industry may be reluctant to sponsor trials in the developing world because the prospects for profit are limited, even if effective treatments are developed. ${ }^{15-17}$ Not for profit organisations may also have difficulty supporting such research on their own. They may have difficulty in setting priorities ${ }^{18}$ and often prefer to offer practical help rather than foster research.

Despite these limitations, the major health problems of the developing world cannot be set aside. Their consequences also affect the developed world. ${ }^{19}$ Success can often be obtained with limited funds. Many diseases in sub-Saharan Africa coexist in vulnerable patient groups, and treatment or prevention of one disease may have a major effect on another (for example, HIV and tuberculosis). ${ }^{20}$ Seemingly expensive interventions can become affordable if there is strong political will and collaboration with the industry.

The pace of clinical research is accelerating in subSaharan Africa. The area needs more international support, but this should be provided without fostering just another form of colonisation. ${ }^{21}$ African researchers should have a meaningful say in setting research priorities, ${ }^{22}$ and outside support should help develop sustainable local research capacity.

We thank Professor Joseph Lau for support and encouragement. We also thank Priscilla Chew and M Perez for help in retrieving articles.

Contributors: The original idea for correlating DALYs and randomised trials belongs to JPAI, who is also the guarantor of the paper. JPAI and PI wrote the first draft of the study protocol, which was edited by GHS and JV. The idea for creating a comprehensive registry of controlled trials for Africa first originated from GHS, EP, and JV under the leadership of JV. PI and EP took the lead in creating the randomised trials' database with help from the other authors. JPAI and PI did the statistical analysis and wrote the first draft of the manuscript. The manuscript was edited for important scientific content by the other authors.

Funding: The project was funded in part by an Innovation Grant by the South African Department of Arts, Culture, Science, and Technology to the South African Medical Research Council.

Competing interests: None declared. 
1 Gross CP, Anderson GF, Powe NR. The relation between funding by the National Institutes of Health and the burden of disease. $N$ Engl J Med 1999;340:1881-7.

2 Murray CJL, Lopez AD. Global burden of disease: a comprehensive assessment of mortality and disability from diseases, injuries, and risk factors in 1990 and projected to 2020. Boston, MA: Harvard School of Public Health, World Health Organization, World Bank, 1996.

3 Horton R. North and South: bridging the information gap. Lancet 2000;355:2231-6.

4 Fraser DW. Overlooked opportunities for investing in health research and development. Bull WHO 2000;78:1054-61.

5 United Nations Statistical Division. Monthly bulletin of statistics. New York: UN, 1992;XLVI:No 7.

6 Murray CJ, Lopez AD. Alternative projections of mortality and disability by cause, 1990-2020: Global Burden of Disease study. Lancet 1997;349:1498-504.

7 Varmus $H$. Evaluating the burden of disease and spending the research dollars of the National Institutes of Health. $N$ Engl J Med 1999;340:1914-5.

8 Yusuf S, Anand S, MacQueen G. Can medicine prevent war? Imaginative thinking shows that it might. BMJ 1998;317:1669-70.

9 Krug EG, Sharma GK, Lozano R. The global burden of injuries. Am J Public Health 2000;90:523-6.

10 Ioannidis JP. Effect of the statistical significance of results on the time to completion and publication of randomized efficacy trials. JAMA 1998;279:281-6.
11 Easterbrook PJ, Berlin JA, Gopalan R, Matthews DR. Publication bias in clinical research. Lancet 1991;337:867-72.

12 Costello A, Zumla A. Moving to research partnerships in developing countries $B M J$ 2000;321:827-9

13 Olkin I. Meta-analysis: reconciling the results of independent studies. Stat Med 1995;14:457-72.

14 Logie ED, Benatar RS. Africa in the 21st century: can despair be turned to hope? BMJ 1997;315:1444-6.

15 Taylor D. The pharmaceutical industry and health in the third world. Soc Sci Med 1986;22:1141-9.

16 Wolffers I, Adjei S, Drift R. Health research in the tropics. Lancet 1998;351:1652-4.

17 Trouiller P, Olliaro PL. Drug development output: what proportion for tropical diseases? Lancet 1999;354:164.

18 Fraser DW. Overlooked opportunities for investing in health research and development. Bull WHO 2000;78:1054-61.

19 Walt G. Globalisation of international health. Lancet 1998;351:434.

20 Msamanga GI, Fawzi WW. The double burden of HIV infection and tuberculosis in sub-Saharan Africa. N Engl J Med 1997;337:801-8.

21 Horton R. Development aid: manna or myth? Lancet 2000;356:1044-5

22 Lee K, Mills A. Strengthening governance for global health research. BMJ 2000;321:775-6.

(Accepted 12 October 2001) 